\title{
ZOONOSES AND HUMAN BEINGS
}

Zoonoses are diseases common to both men and animals. In a simpler way, they may be defined as the diseases transmitted from animals to human beings with participation of domestic and wild animals.

Transmission to man may occur directly, by the contact with infected animals, which eliminate the agent by their secretions; and also indirectly, with ingestion of contaminated water and animals. Participation of vectors and interaction with environment may also occur. For this reason, food of animal origin such as meat, milk and its derivatives, eggs, and honey are of great importance. In several situations, food of vegetal origin must also be considered, for example, in cysticercosis and toxoplasmosis transmission by environmental contamination with consequent spread of the agent.

There are more than 150 diseases of zoonotic character, and some of them are of major importance in public health, for example, rabies, leptospirosis, toxoplasmosis, leishmaniasis, cutaneous and visceral larva migrans, Chagas disease, spotted fever, and others. The incidence of these diseases is high in developing countries since social and economic aspects are practically the determinants to their maintenance and spread. Environmental aspects, such as basic sanitation, besides contributing to the occurrence, make the control of these zoonoses difficult by allowing the agent dissemination into rain water, streams, and brooks, frequented mainly by children.

The advent of AIDS induced the intensification of many zoonoses because the imunodepression caused by the virus leads to manifestation of many latent diseases. In this case, we must consider tuberculosis and toxoplasmosis. Today these diseases, together with cryptococcosis, cryptosporidiosis, and mycobacteriosis, are the most frequent cause of death in patients with AIDS.

Veterinarians play an important role in multiprofessional teams for the resolution of public health problems. Their knowledge of epidemiology regarding zoonoses and the clinical and control aspects of these diseases in animals may avoid the transmission to human beings. The veterinarians notable insertion in the man-animal interface make their responsibility even greater because besides preventing man contamination by animals, these professionals must interrupt the links of the epidemiologic chain of zoonoses transmission by food.

Hélio Langoni, MSc, PhD, Sanitary Veterinarian Department of Public Health Botucatu School of Veterinary Medicine and Animal Husbandry São Paulo State University, UNESP, Botucatu, SP, Brazil hlangoni@reitoria.unesp.br 